\title{
Interface dynamics in Hele-Shaw flows with centrifugal forces: Preventing cusp singularities with rotation
}

\author{
F. X. Magdaleno, A. Rocco, and J. Casademunt \\ Departament d'Estructura i Constituents de la Matèria, Facultat de Física, Universitat de Barcelona, Avenida Diagonal 647, \\ E-08028 Barcelona, Spain \\ (Received 31 January 2000)
}

\begin{abstract}
A class of exact solutions of Hele-Shaw flows without surface tension in a rotating cell is reported. We show that the interplay between injection and rotation modifies the scenario of formation of finite-time cusp singularities. For a subclass of solutions, we show that, for any given initial condition, there exists a critical rotation rate above which cusp formation is suppressed. We also find an exact sufficient condition to avoid cusps simultaneously for all initial conditions within the above subclass.
\end{abstract}

PACS number(s): 47.20.Hw, 47.20.Ma, 47.15.Hg, 68.10.-m

The dynamics of the interface between viscous fluids confined in a Hele-Shaw cell [1-3] has received attention for several decades from physicists, mathematicians, and engineers. In particular, it has played a central role in the context of interfacial pattern formation [4,5]. As a free boundary problem it has the particular interest that explicit timedependent solutions can often be found in the case with no surface tension [6-9]. As a consequence, the issue of the role of surface tension as a singular perturbation in the interface dynamics has received increasing attention [10-14] for its potential relevance to a broad class of problems. However, to what extent the physics of the real problem (with finite surface tension) is captured, even at a qualitative level, by the known solutions is still poorly understood.

As an initial-value problem, the zero surface tension case is known to be ill-posed [11]. An important aspect related to this fact is that some smooth initial conditions develop finitetime singularities in the form of cusps of the interface [6,7]. After this blow-up of the solution, the time evolution is no longer defined. Generation of finite-time singularities is in itself interesting in connection with other singular perturbation problems in fluid dynamics, such as in the case of the Euler equations. In the present problem, surface tension acts as the natural regulator curing this singular behavior, but unfortunately the problem with surface tension is much more difficult and usually defies the analytical treatment. Motivated by this fact, and inspired by recent experiments on rotating Hele-Shaw cells $[15,16]$, we address here the perturbation of the original free boundary problem by the presence of a centrifugal field. This new ingredient enriches the problem in a nontrivial way but, as first discussed in [17], it still admits explicit integration, so it may provide new analytical insights. Here we will focus on the possibility that rotation, although not fully regularizing the problem, may prevent the emergence of singular behavior in the form of cusps at finite time, thus enlarging the class of exact solutions without surface tension which are potentially relevant to the physically realizable situations.

We study an interface between a fluid with viscosity $\mu$ and density $\rho$ and one with zero viscosity and zero density in a Hele-Shaw cell with gap $b$. The cell can be put in rotation with angular velocity $\Omega$ and fluid can be injected or ejected from the cell through an orifice at the center of rotation, with areal rate $Q$. The cases $Q>0$ and $Q<0$ correspond respectively to injecting or ejecting fluid. As in the traditional HeleShaw problem, the flow in the viscous fluid is potential, $\mathbf{v}$ $=\nabla \phi$, but now with a velocity potential given by $[15]$

$$
\phi=-\frac{b^{2}}{12 \mu}\left(p-\frac{1}{2} \rho \Omega^{2} r^{2}\right) \text {. }
$$

Incompressibility then yields Laplace equation $\nabla^{2} \phi=0$ for the field $\phi$ (but not for the pressure). The two boundary conditions at the interface which complete the definition of the moving boundary problem are the usual ones, namely, the pressure on the viscous side of the interface $p=-\sigma \kappa$, where $\sigma$ and $\kappa$ are respectively surface tension and curvature, and the continuity condition for the normal velocity $v_{n}=\mathbf{n} \cdot \boldsymbol{\nabla} \phi$. The crucial difference from the usual case is in the boundary condition satisfied by the Laplacian field on the interface due to the last term in Eq. (1).

This problem is well suited to conformal mapping techniques [2]. The basic idea is to find an evolution equation for an analytical function $z=f(\omega, t)$, which maps a reference region in the complex plane $\omega$, in our case the unit disk $|\omega| \leqslant 1$, into the physical region occupied by the fluid in the physical plane $z=x+i y$, with the physical interface being the image of the region boundary, $|\omega|=1$. We consider two types of situations, one in which the viscous fluid is inside the region enclosed by the interface, and one in which it is outside. It can be shown [15] that the evolution equation for the mapping $f(\omega, t)$ in the rotating case can be written in a compact form as

$$
\operatorname{Im}\left\{\partial_{t} f^{*} \partial_{\phi} f\right\}=\frac{Q}{2 \pi} \vartheta+\frac{1}{2} \Omega * \partial_{\phi} \mathrm{H}_{\phi}\left[|f|^{2}\right]+d_{0} \partial_{\phi} \mathrm{H}_{\phi}[\kappa],
$$

where $\Omega^{*}=b^{2} \Omega^{2} \rho / 12 \mu, d_{0}=b^{2} \sigma / 12 \mu$, and where we have specified the mapping function at the unit circle $\omega=e^{i \phi}$. The curvature is given by $\kappa=-\operatorname{Im}\left\{\partial_{\phi}^{2} f /\left(\partial_{\phi} f\left|\partial_{\phi} f\right|\right)\right\}$ and the Hilbert transform $H_{\phi}$ is defined by

$$
\mathrm{H}_{\phi}[g]=\frac{1}{2 \pi} \mathrm{P} \int_{0}^{2 \pi} g(\theta) \operatorname{cotan}\left[\frac{1}{2}(\phi-\theta)\right] d \theta .
$$


In Eq. (2), $\vartheta=+1$ and $\vartheta=-1$ correspond to the cases with the viscous fluid respectively inside or outside the region enclosed by the interface.

In this context a class of solutions is defined by a functional form of the mapping which is preserved by the time evolution, allowing for a finite number of time-dependent parameters. Some explicit time-dependent solutions for $f(\omega, t)$ with $d_{0}=0$ but $\Omega^{*} \neq 0$ have been previously reported in Ref. [17]. Here we report additional explicit solutions for the rotating case, which all fit into the general rational form

$$
f(\omega, t)=\omega^{\vartheta} \frac{a_{0}(t)+\sum_{j=1}^{N} a_{j}(t) \omega^{j}}{1+\sum_{j=1}^{N} b_{j}(t) \omega^{j}},
$$

although not any mapping of this form is necessarily a solution. Remarkably, we have found that an important class of solutions of the usual case $\left(\Omega^{*}=0\right)$, which is free of finitetime singularities, namely, the superposition of logarithmic terms [9], is no longer a solution for $\Omega * \neq 0$, while mappings with poles turn out to include solutions for both $\Omega^{*}=0$ and $\Omega^{*} \neq 0$. A more detailed study will be presented elsewhere [18]. The polynomial case ( $b_{j}=0$ for all $j$ 's), which in the nonrotating case $\Omega^{*}=0$ is known to always yield finite-time singularities in the form of cusps [6,7], for $\Omega^{*} \neq 0$ is also a solution [17]. However, we will study in the rest of this Rapid Communication that the scenario of cusp formation in these solutions is modified in a nontrivial way by the presence of rotation.

We focus on the role of rotation in preventing cusp formation in the subclass of polynomial mappings of the form

$$
f(\omega, t)=a_{0}(t) \omega^{\vartheta}+a_{n}(t) \omega^{n+\vartheta} .
$$

For $a_{n} \ll a_{0}$ this describes an $n$-fold sinusoidal perturbation of amplitude $a_{n}$ superimposed on a circular interface of radius $a_{0}$. It is convenient to introduce the dimensionless parameter $\varepsilon=(n+\vartheta) a_{n} / a_{0}$. The range of physically acceptable values of $a_{n}$ and $a_{0}$ is given by the condition $0<\varepsilon<1$ for all $n$. We also introduce a scaled mode amplitude $\delta=a_{0} a_{n}$ which turns out to be useful to characterize the interface instability. To see this, let us first compute the standard linear growth rate. Inserting Eq. (5) into Eq. (2) and linearizing in $a_{n}$, we get,

$$
\frac{\dot{a}_{n}}{a_{n}}=\vartheta n \Omega *-(\vartheta n+1) \frac{Q}{2 \pi a_{0}^{2}}-\frac{d_{0}}{a_{0}^{3}} n\left(n^{2}-1\right) .
$$

The term $-Q / 2 \pi a_{0}^{2}$, independent of both $n$ and $\vartheta$, has a purely kinematic origin, associated with the global expansion (or contraction) of the system. This quantity would be the growth rate of an interface mode which followed the (undistorted) flow field with radial velocity $v=Q / 2 \pi r$ [which in turn would imply $\left.a_{n}(t) a_{0}(t)=\mathrm{const}\right]$. Accordingly, the marginal modes for $\delta$ (which in the rotating case may occur for all $n$ ) will be those for which the flow field is undistorted by the interface perturbation, although such perturbation may grow or decay in the original variables $a_{n}$. In this way, growth or decay of $\delta$ will correspond unambiguously to the stability of the flow configuration with the radial velocity field. In this sense it may be justified to qualify the interface instability as described by $\delta$ as "intrinsic,', as opposed to the "'morphological"' one as described by the amplitude $a_{n}$. In this way the intrinsic growth rate takes the simpler form

$$
\frac{\dot{\delta}}{\delta}=\vartheta n\left(\Omega^{*}-Q^{*}\right),
$$

where we have defined $Q^{*}=Q / 2 \pi a_{0}^{2}$ and have dropped the surface tension term, since hereinafter we will focus on to the zero surface tension case. We introduce the relevant dimensionless control parameter of our problem, expressing the ratio of centrifugal to viscous forces, as

$$
P=\frac{\Omega^{*} 2 \pi R^{2}}{Q}=\frac{\pi \rho b^{2} R^{2} \Omega^{2}}{6 \mu Q}
$$

where $R$ is a characteristic radius of the interface.

Equation (7) clearly exhibits the competing effects of rotation and injection, although their roles are not quite symmetric. In fact, notice that $Q^{*}$, which may have both signs, contains a dependence on $a_{0}$. In practice this means that $Q^{*}$ depends effectively on time. An immediate consequence is that the growth of linear modes is not really exponential [15] and may even be nonmonotonic. The asymmetry between injection and rotation shows up also in the fact that the sign of $Q$ determines which of the two effects dominates asymptotically in time. In fact, for positive injection rate the typical radius of the inner fluid is growing while typical interface velocities are decreasing, so centrifugal forces will dominate at long times. On the contrary, for negative injection rate, typical velocities increase while typical radii decrease, so injection will asymptotically dominate over rotation.

In view of Eq. (7), the most interesting configurations will be those in which $Q>0$, so that injection and rotation have counteracting effects. In the case $\vartheta=+1$ (viscous fluid inside), which was experimentally studied in Ref. [15], rotation is always destabilizing. A positive injection rate in this case tends to stabilize the circular interface. However, for fixed $Q$, $Q^{*}$ will decrease with time, so eventually the interface will reach a radius after which all modes are linearly unstable. It is thus expected that, in this case, the formation of cusps can only be delayed but not avoided [18].

The most interesting case from the point of view of preventing cusp formation is $\vartheta=-1$ and $Q>0$, the usual configuration in viscous fingering experiments. In this case, a small rotation rate will only slightly affect the linear instability, but could eventually stabilize the growth at long times, so it is conceivable to have a nontrivial evolution starting from an unstable interface but not developing finite-time singularities.

As an example, we now study the fully nonlinear dynamics of polynomial mappings. Inserting Eq. (5) into Eq. (2) with $d_{0}=0$ we obtain two ordinary differential equations describing the evolution of $a_{0}(t)$ and $a_{n}(t)$. These can be integrated analytically and yield

$$
\begin{gathered}
a_{0}^{2}(t)+\vartheta(n+\vartheta) a_{n}^{2}(t)=\frac{Q}{\pi} t+k_{0}, \\
a_{0}^{n+\vartheta}(t) a_{n}^{\vartheta}(t)=k_{n} e^{n \Omega^{*} t},
\end{gathered}
$$




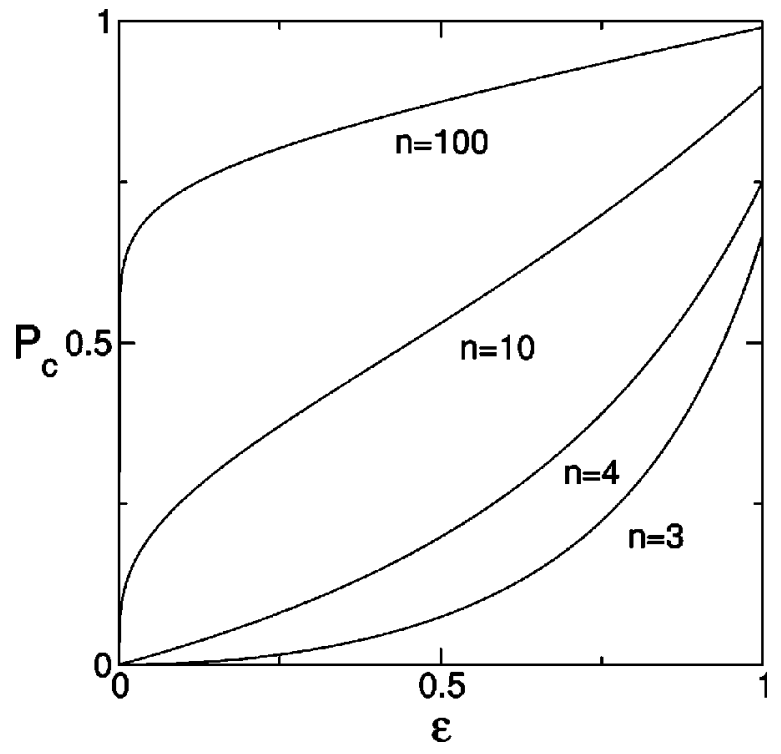

FIG. 1. Critical lines $P_{c}$ for different values of $n$. The region free of cusp singularities for a given $n$ is the one above the correponding curve.

where $k_{0}$ and $k_{n}$ are constants to be determined by initial conditions, and where $n \geqslant 2$ for $\vartheta=+1$ and $n \geqslant 3$ for $\vartheta=$ -1 .

Physically acceptable solutions require that the points in the $\omega$-plane where $\partial_{\omega} f(\omega, t)=0$ (noninvertible) should lie outside the unit disk. The occurrence of a cusp is associated with such a point crossing the unit circle $|\omega|=1$ at a finite time $t_{c}$; that is,

$$
\left|\frac{\vartheta a_{0}\left(t_{c}\right)}{(n+\vartheta) a_{n}\left(t_{c}\right)}\right|=1 \text {. }
$$

If we take the initial value $a_{0}(0)$ as the characteristic length $R$, which coincides with the radius of the perturbed circle if we are in the linear regime, and define the dimensionless time $\tau=\Omega^{*} t$, condition (11) reads

$$
\alpha_{n}\left(\frac{2 R^{2} \tau_{c}}{P}+k_{0}\right)=e^{\beta_{n} \tau_{c}},
$$

where

$$
\alpha_{n}=\frac{(n+\vartheta)^{n / n+2 \vartheta}}{n+2 \vartheta} k_{n}^{-2 /(n+2 \vartheta)}, \quad \beta_{n}=\frac{2 n}{n+2 \vartheta} .
$$

Our aim is now at finding conditions such that an initially smooth interface remains smooth for an infinite time. Thus we have to impose that Eq. (11) should not have any solution for $t_{c}>0$. The transition between the regions with and without cusps will be defined by the conditions that both Eq. (12) and its time derivative are satisfied, such that the curves on each side of Eq. (12) have a common tangent. These two conditions allow us to eliminate $\tau_{c}$, and yield

$$
x \log x-x=-\alpha_{n} k_{0},
$$

where $x=2 \alpha_{n} / P \beta_{n}$, and with $R=a_{0}(0)$ in Eq. (8). We now search for solutions of Eq. (14). For $\vartheta=+1$ it can be proven that this equation has no solutions, and therefore all initial conditions must eventually develop a cusp at finite time, as
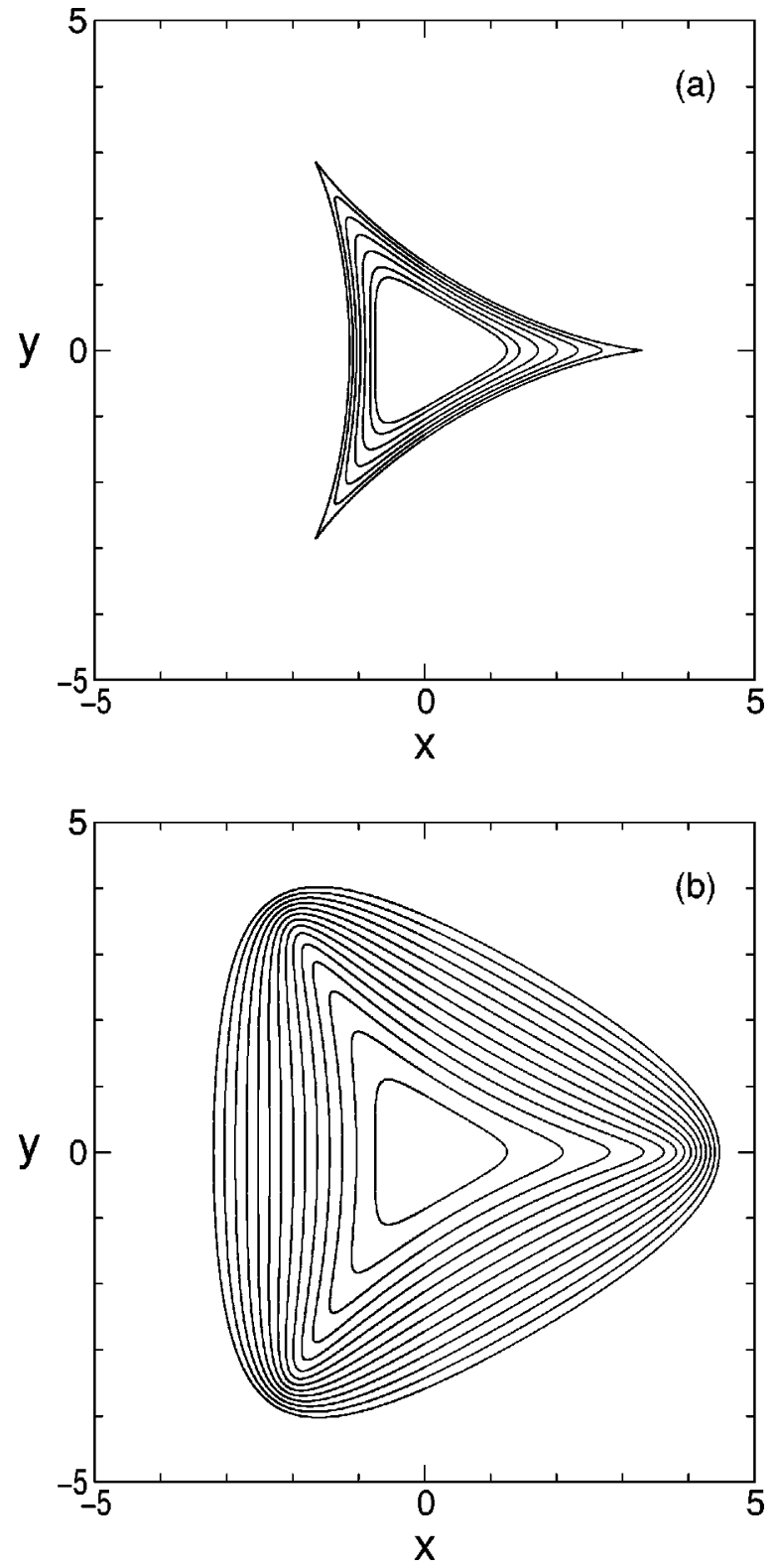

FIG. 2. Evolution of the interface in the case $\vartheta=-1$ (viscous fluid outside), with $n=3, a_{0}(0)=1.0, \epsilon(0)=0.5$, for (a) $\Omega^{*}$ $=0.025$ (cusp formation) and (b) $\Omega^{*}=0.045$ (cusps prevented by rotation).

expected from the linear analysis. On the other hand, for $\vartheta$ $=-1$ the quantity on the rhs of Eq. (14) takes the simple form

$$
\alpha_{n} k_{0}=\frac{n-1}{n-2}\left(1-\frac{\varepsilon^{2}}{n-1}\right) \varepsilon^{2 /(n-2)},
$$

and a nontrivial critical line $P_{c}(\varepsilon ; n)$ can be found for each $n \geqslant 3$. This implies that, in the configuration with the viscous fluid outside, for any initial condition [within the class of polynomial mappings of the form Eq. (5)] there is always a certain rotation rate above which there is no cusp formation. The numerical determination of these curves is shown in Fig. 1.

The leading behavior for initial conditions in the linear regime, $\varepsilon \ll 1$, can be found by expanding the lhs of Eq. (14) around $x=e$ and is given by $P_{c} \approx[(n-1) / n e] \varepsilon^{2 /(n-2)}$. Notice 
that there are qualitative differences for small values of $n$. For $n=3$ the curve starts horizontal at the linear level, implying that a very small rotation rate is sufficient to prevent cusp formation. For $n=4$ the threshold curve starts with a finite slope and for $n>4$ it has an infinite slope at $\varepsilon=0$. A more detailed description and analysis of this diagram will be presented elsewhere [18]. An example of rotation preventing cusp formation is shown in Fig. 2.

In Fig. 1 we also see that for any given $\varepsilon$, the critical $P_{c}$ increases monotonically with $n$. If we take the limit $n \rightarrow \infty$ at fixed $\varepsilon$ we get $\alpha_{n} k_{0} \rightarrow 1$. From Eq. (14) this implies $x=1$ and consequently we obtain an absolute upper bound $P_{c}^{\max }$ $=1$ for all values of $n$ and $\varepsilon$. This implies that, for all initial conditions [within the class Eq. (5)] there is a critical rotation rate

$$
\Omega_{c}=\left(\frac{6 \mu Q}{\pi \rho b^{2} R^{2}}\right)^{1 / 2}
$$

above which cusps are always eliminated. Although Eq. (16) has been derived for the class Eq. (5), with the identification $a_{0}(0)=R$, one might expect that the existence of a certain $\Omega_{c}$ and the scaling with physical parameters given by Eq. (16) could be more general. Notice that $P=1$ corresponds to the intrinsic marginal stability of the circular shape, $Q^{*}$ $=\Omega *$. Therefore, the sufficient condition, valid for all initial conditions of the form Eq. (5), for not developing cusp singularities is that a circular interface with radius given by $a_{0}(0)$ be intrinsically stable, in the sense of Eq. (7). Whether deeper consequences can be drawn in a broader context from this inner connection between the linear problem and the possibility of cusp formation remains an open question.

We acknowledge financial support by the Dirección General de Enseñanza Superior (Spain), Project No. PB96-1001C02-02 and the European Commission Project No. ERB FMRX-CT96-0085.
[1] P. G. Saffman and G. I. Taylor, Proc. R. Soc. London, Ser. A 245, 312 (1958).

[2] D. Bensimon, L. P. Kadanoff, S. Liang, B. I. Shraiman, and C. Tang, Rev. Mod. Phys. 58, 977 (1986).

[3] S. Tanveer, in Asymptotics Beyond All Orders, edited by $\mathrm{H}$. Segur et al. (Plenum Press, New York, 1991).

[4] P. Pelcé, in Perspectives in Physics (Academic Press, New York, 1988).

[5] D. A. Kessler, J. Koplik, and H. Levine, Adv. Phys. 35, 255 (1988).

[6] S. K. Sarkar, Phys. Rev. A 31, 3468 (1985).

[7] S. D. Howison, J. R. Ockendon, and A. A. Lacey, Q. J. Mech. Appl. Math. 38, 343 (1985); S. D. Howison, J. Fluid Mech. 167, 439 (1986).

[8] M. Mineev-Weinstein and S. P. Dawson, Phys. Rev. E 50, R24 (1994); S. P. Dawson and M. Mineev-Weinstein, Physica D 73, 373 (1994).

[9] S. P. Dawson and M. Mineev-Weinstein, Phys. Rev. E 57, 3063 (1998).
[10] W. Dai, L. P. Kadanoff, and S. Zhou, Phys. Rev. A 43, 6672 (1991).

[11] S. Tanveer, Philos. Trans. R. Soc. London, Ser. A 343, 155 (1993).

[12] M. Siegel and S. Tanveer, Phys. Rev. Lett. 76, 419 (1996); M. Siegel, S. Tanveer, and W. S. Dai, J. Fluid Mech. 323, 201 (1996).

[13] F. X. Magdaleno and J. Casademunt, Phys. Rev. E 57, R3707 (1998); F. X. Magdaleno, E. Pauné, and J. Casademunt (unpublished).

[14] M. J. Feigenbaum, I. Procaccia, and B. Davidovich, e-print chao-dyn/9908007.

[15] Ll. Carrillo, F. X. Magdaleno, J. Casademunt, and J. Ortín, Phys. Rev. E 54, 6260 (1996).

[16] Ll. Carrillo, J. Soriano, and J. Ortín, Phys. Fluids 11, 778 (1999); Ll. Carrillo, J. Soriano, and J. Ortín (unpublished).

[17] V. M. Entov, P. I. Etingof, and D. Ya. Kleinbock, Eur. J. Appl. Math. 6, 399 (1995).

[18] A. Rocco, F. X. Magdaleno, and J. Casademunt (unpublished). 